INPLASY

PROTOCOL

To cite: Pan et al. Effectiveness of different types of skin grafting for treating venous leg ulcers: A protocol for

systematic review and network meta-analysis. Inplasy protocol 202130093. doi:

10.37766/inplasy2021.3.0093

Received: 24 March 2021

Published: 24 March 2021

Corresponding author:

Zhangren Yan

zywk20086128@jxutcm.edu.cn

Author Affiliation:

Affiliated Hospital of Jiangxi

University of Traditional

Chinese Medicine

Support: Have support.

Review Stage at time of this submission: Piloting of the study selection process.

Conflicts of interest:

None declared.

\section{Effectiveness of different types of skin grafting for treating venous leg ulcers: A protocol for systematic review and network meta-analysis}

Yan, Z1; Pan, J2; Hu, X3; Yin, H; Zhang, C5.

Review question / Objective: The aim of this Bayesian network meta-analysis of randomized controlled trials is to assess the efficacy of skin grafting for venous leg ulcers systematically.

Condition being studied: Skin grafting is one of the most common and effective treatments for venous leg ulcers. Clinical practice guidelines of the Society for Vascular Surgery and the American Venous Forum recommend that skin grafting and cell-based therapies represent a second-line strategy when a minimum of 4 to 6 weeks of standard wound therapy fail. Nonetheless, insufficient evidence to determine whether other types of skin grafting increased the healing of venous ulcers. Nowadays, with the development of skin grafting technology and cell regeneration medicine, a variety of skin grafting techniques have been applied in clinical practice, especially in the treatment of wound healing. There are still no new studies based on the latest trials and new research methods to evaluate and compare the effect of different types of skin grafting on treating venous leg ulcers. Further studies are required to assess whether other forms of skin grafts increase ulcer healing.

INPLASY registration number: This protocol was registered with the International Platform of Registered Systematic Review and Meta-Analysis Protocols (INPLASY) on 24 March 2021 and was last updated on 31 March 2021 (registration number INPLASY202130093).

\section{INTRODUCTION}

Review question / Objective: The aim of this Bayesian network meta-analysis of randomized controlled trials is to assess the efficacy of skin grafting for venous leg ulcers systematically.
Rationale: Venous leg ulcers are the most common ulcer on the lower extremity, with $4 \%$ of patients over the age of 65 suffering from venous leg ulcers worldwide. As a recurrent, chronic, disabling disease, venous leg ulcers are associated with prolonged disability, important 
socioeconomic impact, and significant psychosocial morbidity. At present, Skin grating is one of the most effective treatments for non-healing ulcers, However, there are still no new studies based on the latest research and new research methods to evaluate and compare the effect of different types of skin grafts for treating venous leg ulcers. Therefore, This study aims to synthesize the available evidence from randomized controlled trails in a network meta-analysis to summarize the best research available, evaluate the effectiveness of different types of skin grafting for treating venous leg ulcers.

Condition being studied: Skin grafting is one of the most common and effective treatments for venous leg ulcers. Clinical practice guidelines of the Society for Vascular Surgery and the American Venous Forum recommend that skin grafting and cell-based therapies represent a secondline strategy when a minimum of 4 to 6 weeks of standard wound therapy fail. Nonetheless, insufficient evidence to determine whether other types of skin grafting increased the healing of venous ulcers. Nowadays, with the development of skin grafting technology and cell regeneration medicine, a variety of skin grafting techniques have been applied in clinical practice, especially in the treatment of wound healing. There are still no new studies based on the latest trials and new research methods to evaluate and compare the effect of different types of skin grafting on treating venous leg ulcers. Further studies are required to assess whether other forms of skin grafts increase ulcer healing.

\section{METHODS}

Search strategy: Studies search will conduct on the following electronic databases: Cumulative Index to Nursing and Allied Health Literature (EBSCO CINAHL Plus), the Cochrane Library, Web of Science, PubMed, Ovid MEDLINE; Ovid EMBASE, China BioMedical Literature (CBM), China National Knowledge Infrchrome1astructure (CNKI), and Wanfang database. In addition, we will search clinical trials registries: the Cochrane Central Register of Controlled Trialsx, the Cochrane Wounds Specialised Register, US National Institutes of HealthOngoing Trials Register Clinical Trials, World Health Organization (WHO) International Clinical Trials Registry Platform (ICTRP), EU Clinical Trials Register. All the databases will be searched from their inception until June 2021, without restrictions for language, or publication on status. The search strategy mainly includes Medical Subject Headings (MeSH) and free-text terms,Such as"Leg U l cer + " [M es H Terms], "Skin Transplantation"[MeSH Terms], "varicose ulcer", "venous ulcer*", "pinch graft*", "bioengineered skin", "randomized controlled trial".

Participant or population: We will include Patients in any care setting with venous leg ulcers. No further restrictions will be made on participants' age, gender, ethnicity and nationality. Methods to diagnose venous leg ulcers wound may vary and this review will accept any as described by the included the studies.

Intervention: The primary intervention was skin grafts or skin replacements applied to venous leg ulcers. We included studies which compared the following types of grafts with any other intervention: (1) pinch grafts (autografts), (2) split-thickness grafts (autografts), (3) full-thickness grafts (autografts and xenografts), (4) cultured keratinocytes/epidermal grafts (allografts and xenografts), artificial skin, bioengineered skin equivalents (allografts and xenografts).

Comparator: The control group was treated with any other intervention.

Study designs to be included: We will include published and unpublished Randomized controlled trials (RCTs). No date or language restrictions will be applied.

Eligibility criteria: We will include published and unpublished randomized controlled 
trails involving participants in any care setting with venous leg ulcers. No further restrictions will be made on participants' age, gender, ethnicity and nationality. The primary intervention was skin grafts or skin replacements applied to venous leg ulcers.

Information sources: Studies search will conduct on the following electronic databases: Cumulative Index to Nursing and Allied Health Literature (EBSCO CINAHL Plus), the Cochrane Library, Web of Science, PubMed, Ovid MEDLINE; Ovid EMBASE, China BioMedical Literature (CBM), China National Knowledge Infrchrome1astructure (CNKI), and Wanfang database. In addition, we will search clinical trials registries: the Cochrane Central Register of Controlled Trialsx, the Cochrane Wounds Specialised Register, US National Institutes of HealthOngoing Trials Register Clinical Trials, World Health Organization (WHO) International Clinical Trials Registry Platform (ICTRP), EU Clinical Trials Register. All the databases will be searched from their inception until June 2021, without restrictions for language, or publication on status.

Main outcome(s): The main outcomes of this review are complete wound healing. A trial had to report at least one of the following as providing the most relevant measures of outcome for the analyses: objective measures of healing (Change or rate of change in wound size, with adjustment), time to complete healing, the proportion of ulcers healed within the trial period, as defined by the trial authors, recurrence of VLUs (as reported in the trial).

Additional outcome(s): Additional outcomes included: Health-related quality of life; pain; adverse events; costs withdrawals and acceptability of treatment.

Data management: We will export the documents retrieved from the database to EndNote $X 9$ software and will use it to manage and delete duplicates. We will establish the document information extraction table in pre-designed Excel including the following information from each included study: article title, author, publication time, demographic characteristics of the subjects, sample size, allocation method, interventions in the treatment group and control groups, course of treatment, the severity of disease, adverse events, data analysis strategy, and outcome indicators.

Quality assessment / Risk of bias analysis: Two review authors will independently use Cochrane's Risk of Bias tool to appraise the risk of bias of each included study. Any discrepancy between two reviewers will be resolved by discussion and a third reviewer where necessary.

Strategy of data synthesis: Data will be synthesized with a pairwise meta-analysis in a frequentist framework by Review Manager (RevMan version 5.3). The Bayesian network meta-analysis will be conducted in the WinBUGS software(Version 1.43, Medical Research Council Biostatistics Unit, Cambridge, UK) and STATA software(Version 14.2). And the Markov Chains Monte Carlo simulation technique will be used to generate samples.

Subgroup analysis: According to the different sources of heterogeneity, subgroup analysis can be carried out, such as treatment time, course of disease, and age.

Sensitivity analysis: Sensitivity analysis will be carried out at the test results with obvious heterogeneity, such as treatment time, course of the disease, basic disease, gender, age, and so on.

Language: No restrictions for language.

Country(ies) involved: This systematic review and network meta-analysis will be conducted in China.

Keywords: venous leg ulcers, skin grafting, network meta-analysis, randomized controlled trials. 
Dissemination plans: The results of the study will be submitted to a peer-reviewed journal for publication.

Contributions of each author:

Author 1 - Junqing Pan - The author drafted the manuscript.

Email: panjunqing2020@gmail.com

Author 2 - Xiangjun $\mathrm{Hu}$ - The author provided statistical analysis.

Email: 980500393@qq.com

Author 3 - Hongwei Yin - The author contributed to the development of the selection criteria, data extraction, and the risk of bias assessment strategy.

Email: 2450479648@qq.com

Author 4 - Congzhong Zhang - The author contributed to the development of the selection criteria, data extraction, and the risk of bias assessment strategy.

Email: 1634559657@qq.com

Author 5 - Zhangren Yan - The author read, provided feedback and conceptualization, and approved the final manuscript.

Email: zywk20086128@jxutcm.edu.cn 